\title{
Addressing the Challenges in dental practice during Covid-19: A review
}

\author{
Sumeet Gupta' , Swati Gupta2* , Rohan D Jagtap ${ }^{3}$ \\ 'Dentist and research scholar, Panjab University, Chandigarh, India \\ ${ }^{2}$ Periodontist, Gainesville, $\mathrm{Fl}$, USA \\ ${ }^{3}$ Director, Division of Oral and Maxillofacial Radiology, Assistant Professor, Department of Care Planning and Restorative Sciences \\ University of Mississippi Medical Centre School of Dentistry, USA
}

Keywords: Covid-19, dentistry, guidelines, healthcare

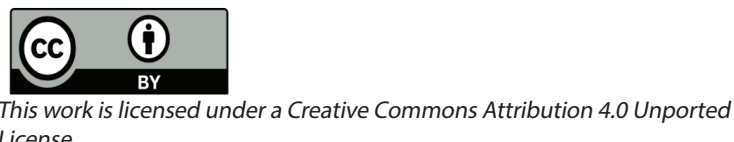
License.

\section{Introduction}

Coronaviruses, named after the crown of spikes, they carry on their surface are single stranded RNA viruses belong to the family of Coronaviridae. ${ }^{1}$ So far, 4 different types of coronaviruses namely- SARS (SARS-CoV), MERS (MERS Cov), Coronavirus infecting birds and mammals causing gastroenteritis, and most recently SARS- Cov-2, are known worldwide. ${ }^{2}$ Coronaviruses have been etiologically known to cause respiratory illness in human and other mammals. ${ }^{3}$ SARS-CoV-2, even though claimed to be less deadly than SARS and MERS, is highly contagious and spreads faster. The mortality rate, variable in various countries, so far on an average is 6-10 times more than a seasonal influenza, with $10-27 \%$ in patients aged $>85$ years to $<1 \%$ in adults aged 20-54 years. ${ }^{4}$ Patients suffering from Covid-19 can be either asymptomatic for a period of at least 14 days, owing to the 6-12 day incubation period of the virus or present with most common flu like symptoms fever and dry cough( 48-92\%), shortness of breath, fatigue and less common symptoms of headache, pharyngitis, productive cough anosmia and gastrointestinal symptoms.[2] Regarding the immunopathogenesis of the viruses, three different settings have been described ${ }^{5}$ :

1. Paucisymptom patient: Patients with high viral titre in nasopharyngeal secretions as well as faeces.

2. Decompensation phase: Low viral titre compared in nasopharyngeal secretions

3. Progression and death: Persistent viremia and high viral titres in upper and lower respiratory tract.

\section{Abstract}

Covid- 19 was declared as global pandemic by WHO. World is not unknown to the family of coronaviruses due to previous epidemic caused by SARS and MERS. Healthcare professions have from time to time change their ways of practicing because of the direct threat posed by such infections, capable of causing human to human transmission. This article, based on our experience and guidelines issued by CDC as well as IDA, intends to provide a review of recommended protocols for dental professionals.
So far, with no vaccine available, few drugs like Hydroxychloroquine, Liponavir, Oseltamivir, Ribavirin, Remdesivir are under clinical trials. ${ }^{6-8}$ Hospitals are providing supportive care comprising of oxygen and ventilator to the affected individuals. Worldwide lockdown of all non-essential businesses was issued in early March and April, with many healthcare practices including dentistry limited to delivering urgent care only. Dental practices, which were limited to only emergency care, will resume addressing the issues of a huge number of patients in near future. This brings us to a question "How to avoid the risk of transmission in dental clinics?"

\section{Risk to dental professionals}

Dental professionals are at a very high risk of getting exposed to the coronavirus from an asymptomatic patient. This was also highlighted by a New York times article "The Workers Who Face the Greatest Coronavirus Risk." sneeze, droplet inhalation and contact transmission through oral, nasal, and mucous membranes have been proposed to be the most common routes of transmission of the virus. ${ }^{10} \mathrm{~A}$ study done by scientists in USA concluded that virus could survive and remain infectious in aerosol for a period of 3 hours. ${ }^{11}$ Another study from China demonstrated that the transmission distance of SARS-CoV-2

\footnotetext{
${ }^{*}$ Corresponding Author

Dr. Swati Gupta

BDS, MDS Periodontics

Private practice, Gainesville, Florida, USA

Email: swati.gup9@gmail.com
} 
might be $4 \mathrm{~m} .{ }^{12}$ The same study also demonstrated that the virus was widely distributed on sickbed handrails, computer mice and floors. The possible transmission routes in dental practice come from:

1.Direct contact with the asymptomatic/ symptomatic patient: Contact transmission via eye, oral and nasal mucous membranes. [10] Studies have proven that SARS-CoV-2(2019- nCov), like SARS, use AEC2 as receptors to invade cells and that may promote human to human transmission. ${ }^{13}$ These AEC2 cells are abundantly found in respiratory tract as well as in cells compatible with salivary gland duct epithelium in the mouth. ${ }^{14} \mathrm{~A}$ study had reported that on average sampling day 4.8, SARS-CoV RNA could be detected in both throat wash and saliva. ${ }^{15}$ Many studies have reported the presence of Covid-19 in deep throat saliva. ${ }^{16-18}$

2.Transmission via cough, sneeze, and droplet inhalation

\section{Implications and guidelines for dental practice}

In the wake of this deadly pandemic, every industry had to change their working pattern. Healthcare industry has had to face and probably in future will face long term implications of this outbreak in terms of operational challenges and medical supply disruption. Clinicians will always be in a dilemma since they are obliged to provide services to the society and at the same time ensure the safety of the patients and themselves. Dentists are at huge risk of getting exposed to virus and pathogenic microorganisms due to the specificity of the profession, which includes face to face communication, dealing with saliva, blood, body fluids and handling infected instruments or contaminated surfaces. In the present scenario, it is prudent for all the dental professionals to adopt certain guidelines for everyone's safety. Our recommendations are based on the guidelines proposed by $\mathrm{CDC}^{19}$ and IDA ${ }^{20}$ as well as from our own experience due to the recent outbreak.

1. Tele dentistry: Oral health is an important part of physical health and undeniably, it affects mental health. Patients with systemic conditions like hypertension and diabetes, mental illnesses of any kind rely heavily on periodontal maintenance as well oral health check-ups by the dentists and in the absence of unmet oral health needs, may develop anxiety. ${ }^{21}$ Under the present scenario, it would be best if healthcare providers could establish a protocol and be in touch with their patients either through web chat or phone lines. This will allow the dentist to address patient's needs while limiting exposure at the same time.

2. Patient evaluation: $A$ health professional should be able to identify a patient with suspected case of Covid-19. A through medical history and travel history should be taken from every patient before scheduling any appointments. Patient should be asked the following screening questions:

A.Do you have fever?

B.Have you experienced a recent onset of a respiratory problem, such as a cough or difficulty breathing?

C.Have you within the last 14 days travelled, internationally or visited an area where documented or suspected community transmission of Covid-19 is occurring?

D.Have you come into contact with a patient with Covid-19 in the past 14 days?

E.Have you recently participated in any gathering, meetings, or had close contact with many unacquainted people?
IDA guidelines also suggest to post a sign at the entrance of dental clinics suggesting patients to reschedule their appointments and contact a physician if they have fever, cough, sneezing or shortness of breath. ${ }^{20}$ It has also been seen that $80 \%$ of the patients are asymptomatic and not severe. ${ }^{2}$ To rule out, travel history should be taken and only emergency treatment with recommended barrier protection and hygiene should be provided, if a patient is suspected of having Covid-19.

3. Waiting room hygiene: Visual alerts, signs and posters should be placed in all the strategic places in waiting rooms, elevators etc. Studies have shown that the virus can last from up to 4 hours to 24 hours on plastic and papers ${ }^{11}$ and 9 days on inanimate surfaces. ${ }^{14}$ Hence, to prevent community transmission in the waiting rooms, adequate standards of sanitization should be maintained. All the surfaces like door handles, tables, chairs should be frequently sanitized. For the decontamination of inanimate surfaces, it has been proposed to use a dilution of 1:50 of standard bleach ${ }^{14}$ or $70 \%$ ethanol for small surfaces, as recommended by WHO. ${ }^{22}$ Potential faecal transmission has been reported in a study ${ }^{23}$ and hence it is imperative to maintain hygienic conditions in all the places like bathrooms, potentially used by anyone in the dental clinic to prevent any kind of community transmission. Hand sanitizers should be made available in waiting rooms. Another potential way to prevent community transmission would be to not allow many patients in waiting room and follow the protocol of social distancing. Precautions should be taken with the air conditioning systems.

4. Personal Protection: Hand hygiene protocols as recommended by CDC should be followed. Hands should be washed before and after any procedure with $60-95 \%$ alcohol or soap and water for 20 seconds. ${ }^{19}$ Protective gowns, masks, gloves should be worn by every health personnel in the clinic and should be changed when soiled. Gloves and mouth masks should be changed after each patient to prevent any cross contamination.

5. Protection during dental procedures: A preprocedural mouth rinse with $1 \%$ hydrogen peroxide or $0.2 \%$ povidone has been recommended to reduce viral load. It has been suggested by a study that chlorhexidine might not be very effective against this strain of coronavirus. ${ }^{24}$ Rubber dams have been suggested to reduce airborne particles in 3 -foot diameter of the operational field by $70 \%{ }^{25}$ and hence should be used especially in procedures involving high speed handpieces and ultrasonic devices. Hand scaling or mechanical devices should be used for removal of caries. Hand pieces with anti-retraction valves should only be used. Anti-retraction hand pieces prevent the backflow of bacteria and virus into the tubes of handpiece and dental unit. ${ }^{26}$ WHO has recommended that any procedure that induces coughing should be avoided. ${ }^{27}$ Taking intraoral radiograph is one such procedure, hence probably it would be best if clinicians could use extraoral radiographic modalities like panoramic and CBCT.

\section{Conclusion}

Coronavirus pandemic has challenged every sector of the society to mend their ways of functioning but in particular healthcare profession, by far, is the most impacted since the professionals have to deal with the symptomatic patients to provide emergency care or asymptomatic patients for routine care. In this commentary article, we have tried to suggest ways to minimize the transmission of infection in dental clinics. Dental professionals should constantly update their knowledge and be aware of any 
impending infectious threats posed by such infectious diseases.

\section{Take home message}

Covid-19 is a viral deadly disease and every precaution should be taken to prevent the transmission of the disease.

Healthcare professionals have a duty to provide service to the society but at the same time to ensure safety to them and the patients.

Dentists should ensure their dental clinics are well-equipped and updated as per CDC guidelines.

All healthcare professional should keep themselves updated with the new information pertaining to Covid-19.

Conflict of interest: Nil

Funding: Nil

\section{Publication History:}

EIC: Umid Kumar Shrestha, Nepal Mediciti, Lalitpur, Nepal

Date of submission: May 10, 2020

Date of acceptance: May 22, 2020

\section{Reference}

1. Bradburne AF, Tyrrell DAJ. Coronaviruses of man. Prog Med Virol $1971 ; 13: 373-84$.

2. Auwaerter, Paul G. "Coronavirus COVID-19 (SARS-CoV-2)." Johns Hopkins ABX Guide, The Johns Hopkins University, 2017. Johns Hopkins Guide, www.hopkinsguides.com/hopkins/view/Johns Hopkins_ABX_Guide/540747/all/Coronavirus_COVID_19_SARS_ CoV_2_.

3. Fields BN, Knipe DM, eds. Fields virology. 2nd ed. New York: Raven Press; 1990.

4. CDC COVID-19 Response Team. Severe Outcomes Among Patients with Coronavirus Disease 2019 (COVID-19) - United States, February 12-March 16, 2020. MMWR Morb Mortal Wkly Rep. 2020; 69:343-6. doi: http://dx.doi.org/10.15585/mmwr.mm6912e2.

5. Lescure FX, Bouadma L, Nguyen D, Parisey M, Wicky PH, Behillil $\mathrm{S}$, et al. Clinical and virological data of the first cases of COVID-19 in Europe: a case series. Lancet Infect Dis. 2020; S1473-3099:302000. [Epub ahead of print]

6. Cao B, Wang Y, Wen D, Liu W, Wang J, Fan G, et al. A Trial of Lopinavir-Ritonavir in Adults Hospitalized with Severe Covid-19. N Engl J Med 2020. [Epub ahead of print]

7. Gao J, Tian Z, Yang X. Breakthrough: Chloroquine phosphate has shown apparent efficacy in treatment of COVID-19 associated pneumonia in clinical studies. Biosci Trends. 2020; 14:72-3.

8. Jun C, Danping L, Li L, Ping L, Qingnian X, Lu X, et al. A pilot study of hydroxychloroquine in treatment of patients with common coronavirus disease-19 (COVID-19). J Zhejiang Univ (Med Sci) 2020; 49: 0-0.
9. The workers who face the greatest coronavirus risk. The New York Times (New York) 2020 March 15. Available from https://www.nytimes.com/interactive/2020/03/15/business/ economy/coronavirusworker-risk.html. [Last accessed on April19, 2020].

10. Lu CW, Liu XF, Jia ZF. 2019-nCoV transmission through the ocular surface must not be ignored. The Lancet 2020;395: e39.

11. Van Doremalen N, Bushmaker T, Morris DH, Holbrook MG, Gamble A, Williamson BN, et al. N Engl J Med 2020; 382:15647.

12. Guo Z-D, Wang Z-Y, Zhang S-F, Li X, Li L, Li C, et al. Aerosol and surface distribution of severe acute respiratory syndrome coronavirus 2 in hospital wards, Wuhan, China, 2020. Emerg Infect Dis 2020; 10;26. [Epub ahead of print]

13. De WE, Van DN. Falzarano D, Munster VJ. SARS and MERS: recent insights into emerging coronaviruses. Nat. Rev. Microbiol 2016; 14: 523-34.

14. Kampf G, Todt D, Pfaender S, Steinmann E. Persistence of coronaviruses on inanimate surfaces and its inactivation with biocidal agents. J. Hosp. Infect 2020; 104:246-51.

15. Wang WK, Chen SY, Liu IJ, Chen YC, Chen HL, Yang CF, et al. Detection of SARS-associated coronavirus in throat wash and saliva in early diagnosis. Emerg Infect Dis. 2004; 10:1213-9.

16. To KK, Tsang OT, Chik-Yan Yip C, Chan KH, Wu TC, Chan JMC, et al. Consistent detection of 2019 novel coronavirus in saliva. Clin. Infect. Dis.2020 [Epub ahead of print]

17. Zhang W, Du RH, Li B, Zheng XS, Yang XL, Hu B, et al. Molecular and serological investigation of $2019-n C o V$ infected patients: implication of multiple shedding routes. Emerg. Microbes Infect 2020; 9, 386-9.

18. Chen L, Zhao J, Peng J, Li X, Deng X, Geng Z, et al. Detection of 2019-nCoV in Saliva and Characterization of Oral Symptoms in COVID-19 Patients. Available at SSRN: https://ssrn.com/ abstract $=3556665$.

19. Interim Infection Prevention and Control Recommendations for Patients with Suspected or Confirmed Coronavirus Disease 2019 (COVID-19) in Healthcare Settings. Available at https:// www.cdc.gov/coronavirus/2019-ncov/hcp/infection-controlrecommendations.html.[Accessed on April19,2020].

20. Indian Dental Association's Preventive Guidelines for Dental Professionals on the Coronavirus Threat. Available at https://www.ida.org.in/pdf/IDA_Recommendations_for_Dental_ Professionals_on_the_Coronavirus_Threat.pdf. [Accessed on April $19,2020]$

21. Kisely S. No Mental Health without Oral Health. Can J Psychiatry 2016; 61:277-82

22. WHO. Annex G. Use of disinfectants: alcohol and bleach. Infection prevention and control of epidemic-and pandemicprone acute respiratory infections in health care. Geneva: WHO; 2014. p. $65 \mathrm{e} 6$. 
23. Gu J. COVID-19: Gastrointestinal manifestations and potential fecal-oral transmission. Gastroenterol 2020. [Epub ahead of print]

24. Peng $X$, X U X, LiY, Cheng L, Zhou X, Ren B. Transmission routes of 2019-nCoV and controls in dental practice. Int J Oral Sci 2020;12: 9.

25. Samaranayake LP, Reid J, Evans D. The efficacy of rubber dam isolation in reducing atmospheric bacterial contamination. ASDC J Dent Child 1989;56: 442-4.

26. Hu T, Li G, Zuo Y, Zhou X. Risk of hepatitis B virus transmission via dental handpieces and evaluation of an anti-suction device for prevention of transmission. Infect Control Hosp Epidemiol 2007; 28: $80-2$.

27. World Health Organization. 2020a. Clinical management of severe acute respiratory infection when novel coronavirus (2019$\mathrm{nCoV}$ ) infection is suspected: interim guidance. Available at https://www.who.int/ publications-detail/clinical-managementof-severe-acute-respiratory-infection-when-novel-coronavirus(ncov)-infection-is-suspected. [Accessed on April 19,2020]. 